\title{
Virtual Testing Method of Human Knee Prosthesis
}

\author{
Z. KHEIREDDINE ${ }^{1}$, I. OLDAL ${ }^{2}$ \\ 1PhD student, Faculty of Mechanical Engineering, Szent István University \\ E-mail: khaireddinezehouani093@gmail.com \\ 2Faculty of Mechanical Engineering, Szent István University \\ E-mail: oldal.istvan@gek.szie.hu
}

Abstract. In the human knee joint, a degenerative wear of the joint can be formed, the common treatment of this disease is the total knee prosthesis geometry replacement and prosthesis implanting, the aim of our research is the study of the human knee joint with applications in prosthesis, we will develop a method by creating a virtual knee prosthesis geometries, we will generate the geometries using the ADAMS program and examined on a special knee prosthesis qualification equipment.

Keywords. Biomechanics, Knee prosthesis, ADAMS program, knee

\section{Introduction}

The human knee joint is the biggest and most complicated joint of the human body, but it is also very vulnerable. Due to joint disorders (mainly gonarthrosis), it is necessary to install a prosthesis in the knee joint. In many cases, they need to be replaced over time due to loosening or worn out prosthesis. In order to have the kinematic characteristics of the prosthesis as close as possible to the healthy knee joint and to make it less necessary to exchange them. The kinematic joint needs to be known, in the knowledge of kinematics, knee people are researching the kinematic joint, however, the methods and evaluations used by the researchers different from many perspectives, so their results cannot be compared, accordingly there is no generally accepted articular kinematic.
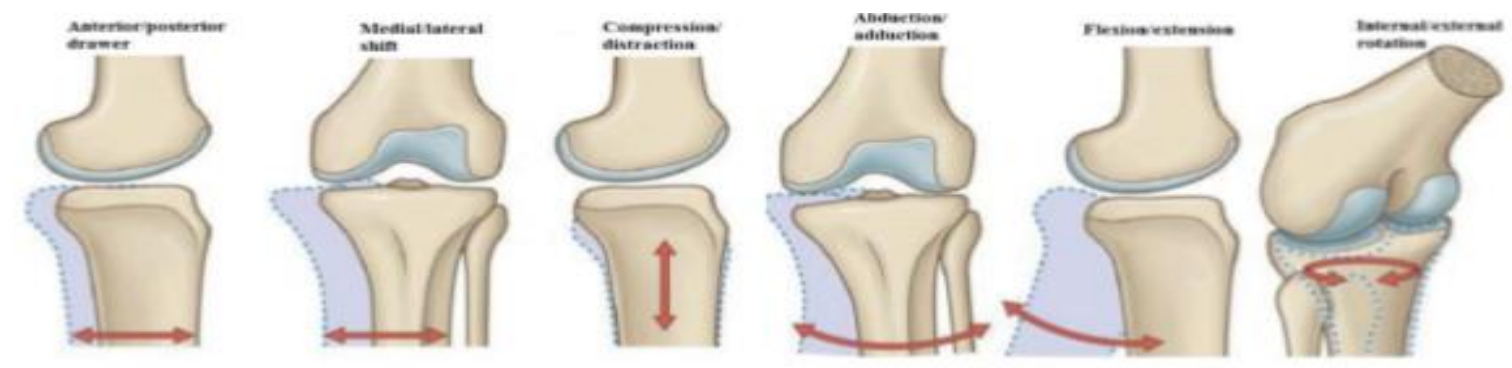

Figure 1. knee joint motion in three dimensions, which is described using six independent variables (Standring, 2008)

The knee is the largest and most complicated joint in the human body (Bert, 2005), (Shi, 2007). The knee is a joint connecting the femur (thigh bone), patella (knee cap) and the tibia (shin bone). The 
fibula, connected to the tibia, also forms part of the knee joint. Figure 1 shows the main components of the knee joint. In humans, the knees support almost the entire weight of the body and are therefore very vulnerable to injury and to the development of osteoarthritis.

Osteoarthritis is the abnormal wearing of the cartilage that covers the joints, as well as the decrease of synovial fluid, which acts as lubricant for the joints. This results in low-grade inflammation of the joints, which leads to pain and can greatly affect the quality of life of a person. For instance, simple tasks like walking or climbing stairs can become difficult. Movement of the knee can be separated into the two major articulations within the knee, the tibiofemoral articulation and the patellofemoral articulation.

For the development of knee prosthesis geometry it is essential to know the movement of the real human knee joint. The Biomechanical Research Group of the Szent István University determined the specific rotation-flexion function of the human knee joint (Fig. 2.). The movement was with three linear functions approximated as the movement has three main phases. That function is the so called reference function (Katona et al, 2013). For a prosthetic geometry development, this is used such as input parameter to be achieved. The research group has developed for the cadaver machine a knee prosthesis measuring and qualification system. In this case the new prosthesis geometry can be adequately tested and compared with the cadaver results.

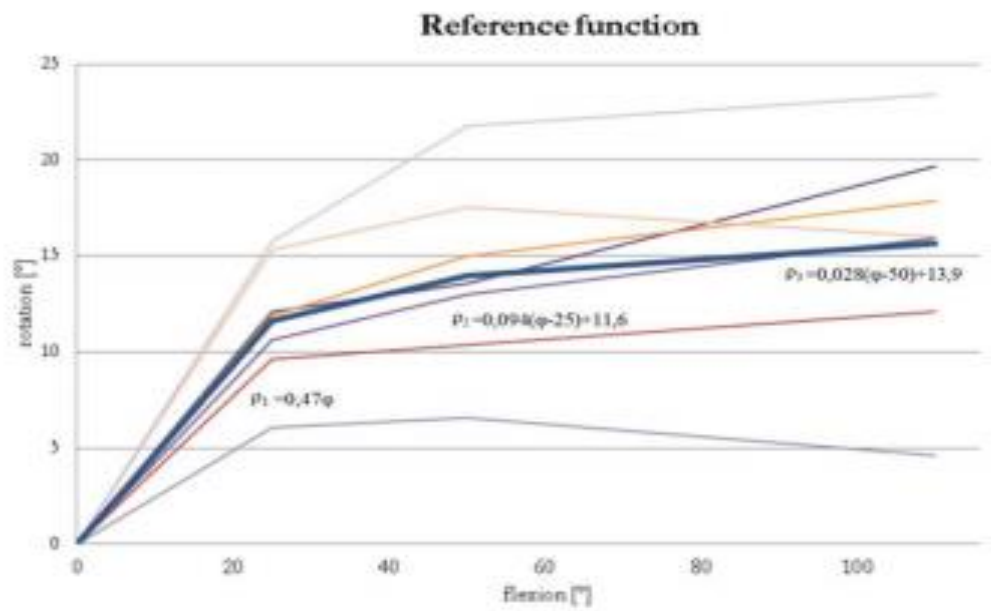

Figure 2. The results of different authors (Katona et al, 2013)

Additional reasons for the differences between the curves are the followings: biological differences between the people, the applied experimental apparatus, the method of measurement and evaluation, different types of motion and at last the measurement error. The separation of these reasons is an important task in order to draw general conclusions. The diversity of apparatuses and the evaluation methods have significant role in the differences between the results. The upcoming errors can be significantly reduced by using simple apparatus for the experiments. For this reason, I will be using a simple experimental device developed by a research team at Szent István University.

This apparatus helps the error analysis, the comparison of the measurements and ensures the unconstrained movement of the knee joint. One of the aims was to obtain precise and reproducible kinematical data possible about the knee joint motion. General aim of the experimental apparatus design and construction was to carry out experiments on cadaver knee joints, in order to provide 
kinematic data to the knee prosthesis design. The apparatus was later further modified in a way that even prostheses can be clamped into the same apparatus, thus experiments under the same conditions can be carried out in order to compare the results of the measurements with the physiological joint kinematics. The usability of the cadaver knee joint as experimental model has only been studied by the default installation of the experimental apparatus in case of one motion type. This motion is the flexion of the tibia with a horizontally fixed femur. The experimental apparatus is multifunctional, which is suitable for the determination of the cadaver knee kinematics and kinetics (Figure 3). It can be used in $\mathrm{MR}$, in case of different type of loads and it is also suitable for testing knee prostheses.

\section{Materials and Methods}

The knee prosthesis is consisting of two components they are the tibia and the femoral component, the so called reference function must be achieved with its adequate geometry.

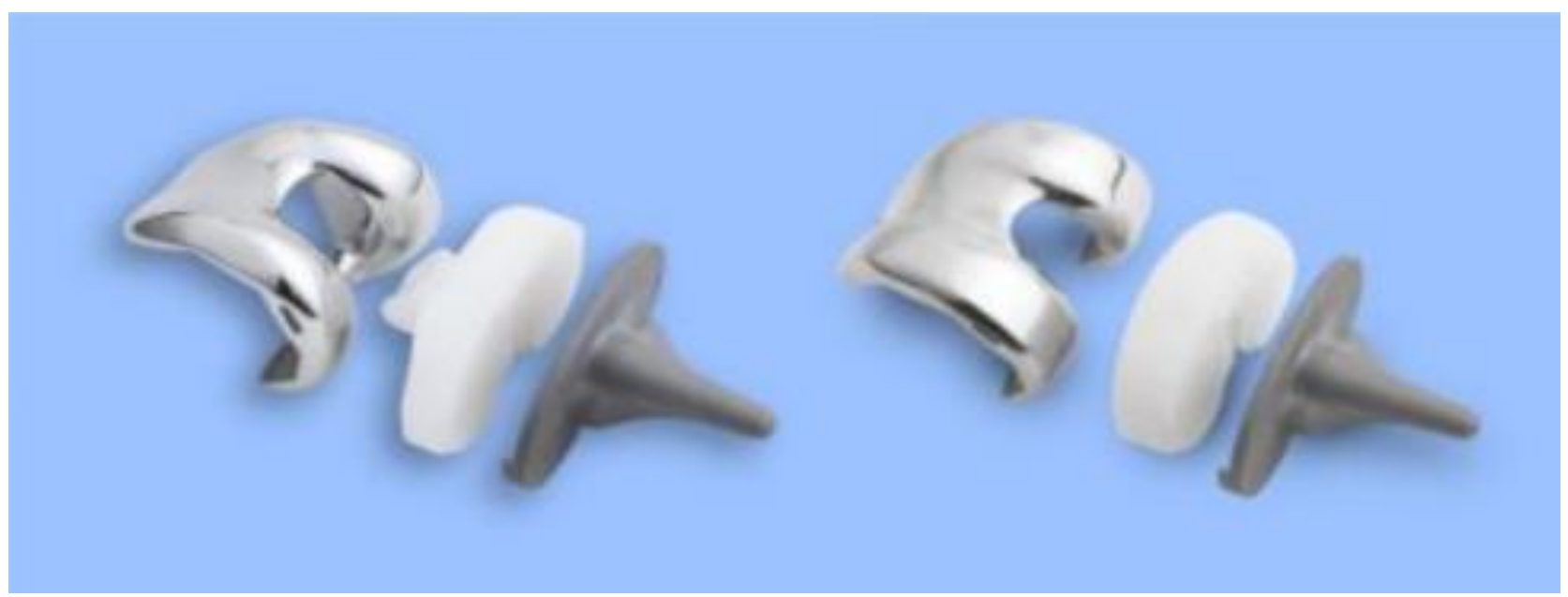

Figure 3. Physical prosthesis for human knee joint.

The measurement performed on the experimental test machine shown in (figure 4), the flexion of the knee prosthesis components is performed by a stepper motor.

The rotation-flexion values are recorded by two incremental rotary encoders with a resolution of $180^{\circ}$.

Due to the high-stepping restore accuracy of the stepper motor, all measurement we will carried out in the same range flexion.

Test machine is multifunctional, which is suitable for the determination of testing knee prostheses (Figure 5). and in case of different type of loads and it is also suitable for the knee kinematics and kinetics. 


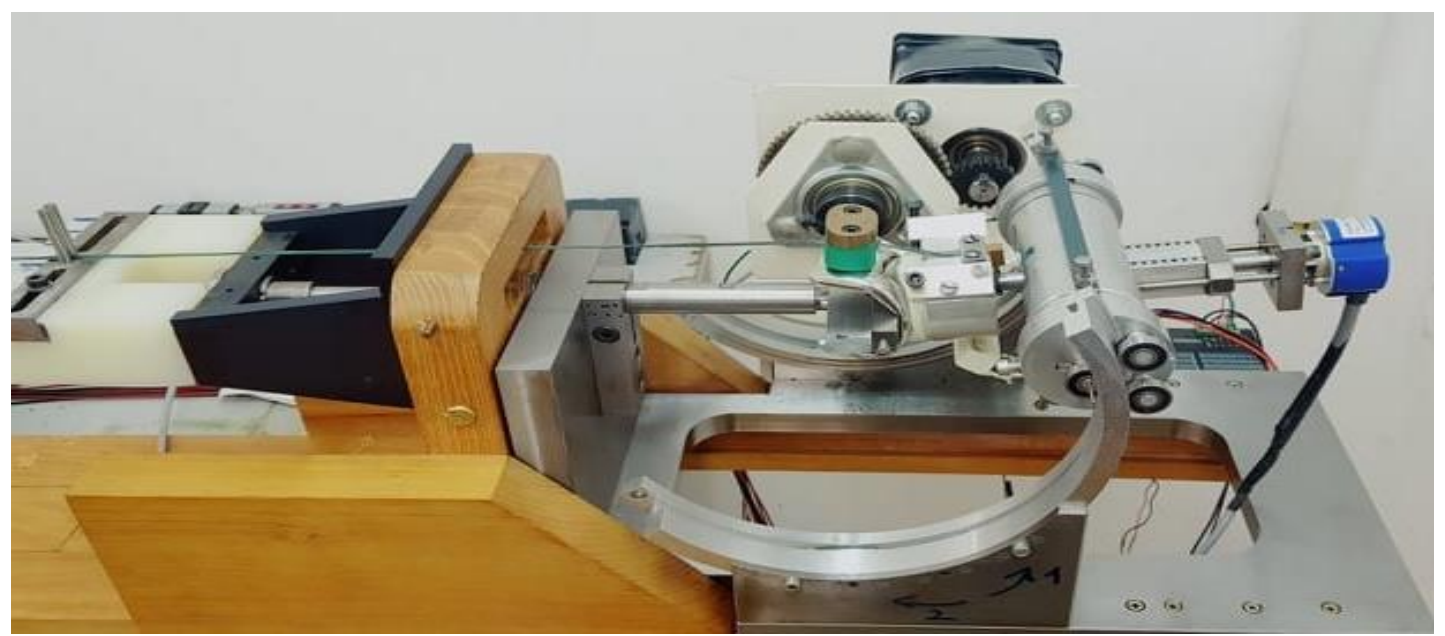

Figure 4. Knee prosthesis test machine.

For the development of knee prosthesis geometry, it is essential to know the movement of the real human knee joint, we will use this machine, so we will study these positions for knee prosthesis.

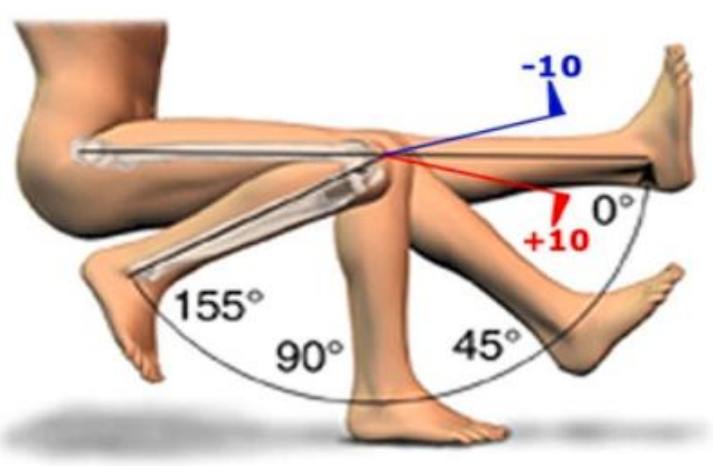

Figure 5.

1- Normal Knee:

Normal range of motion of knee is

Flexion - 120-150 degrees

Extension (-5) to (-10) degrees

Internal rotation with knee flexed - 10 degrees

External rotation with knee flexed - 30-40 degrees

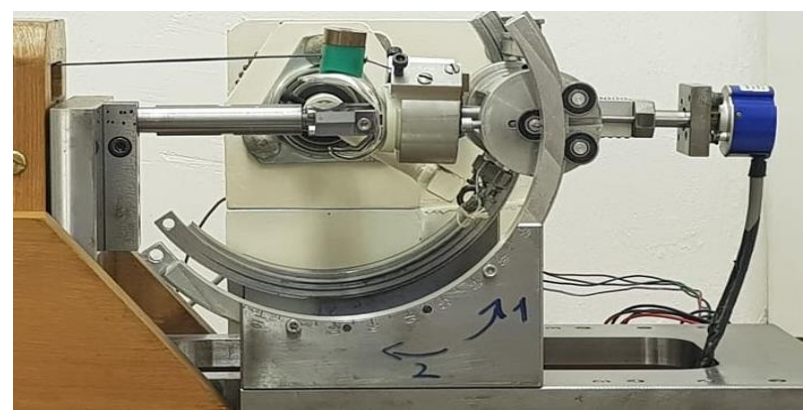

Figure 7. 2) straight knee position with femur and tibia.

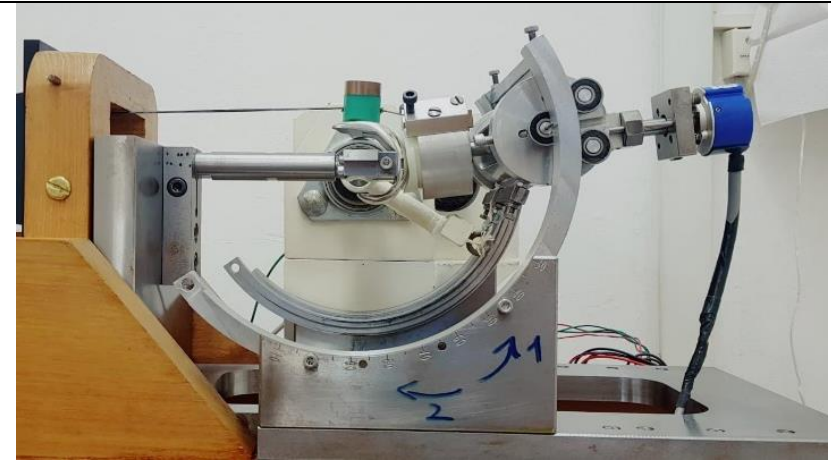

Figure 6.

2-knee prosthesis:

This position Knee extension -10 degrees.

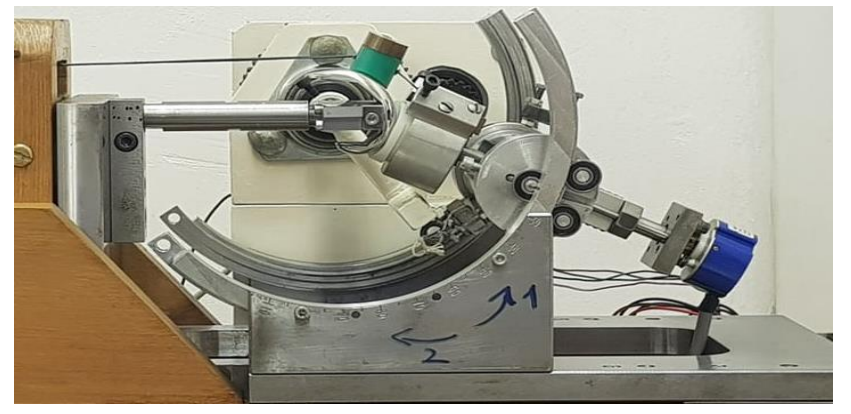

Figure 8. 3) This position Knee flexion 45 degrees 


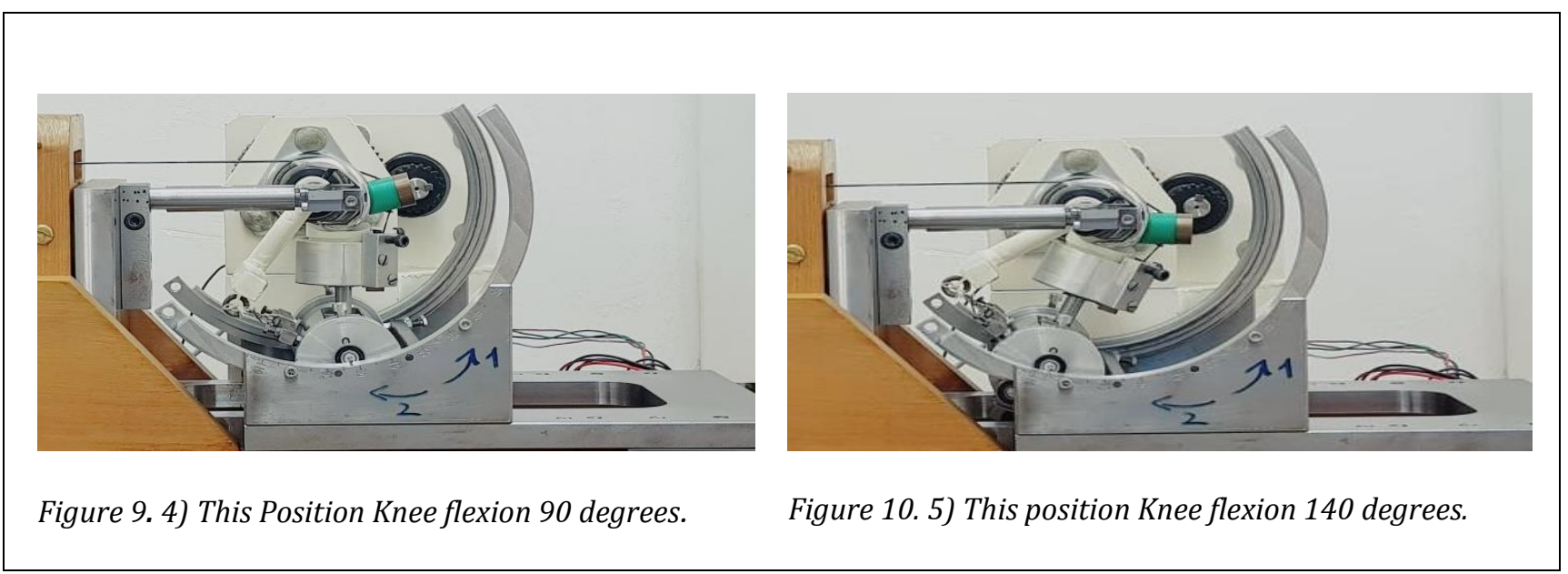

The knee prosthesis is consisting of two components they are the tibia and the femoral component. The so called reference function must be achieved with its adequate geometry. in our research we will begin our investigations based on available knee prosthesis geometry (Fig. 12.).

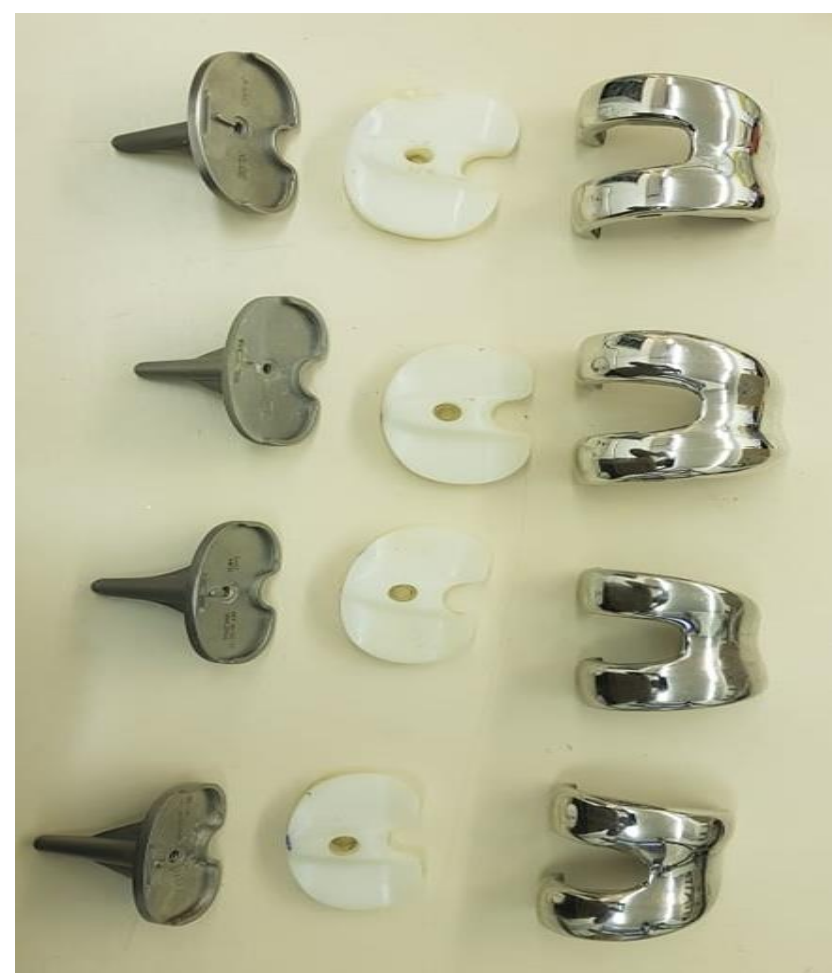

Figure11. 4 types of the knee prosthesis(cadaver)

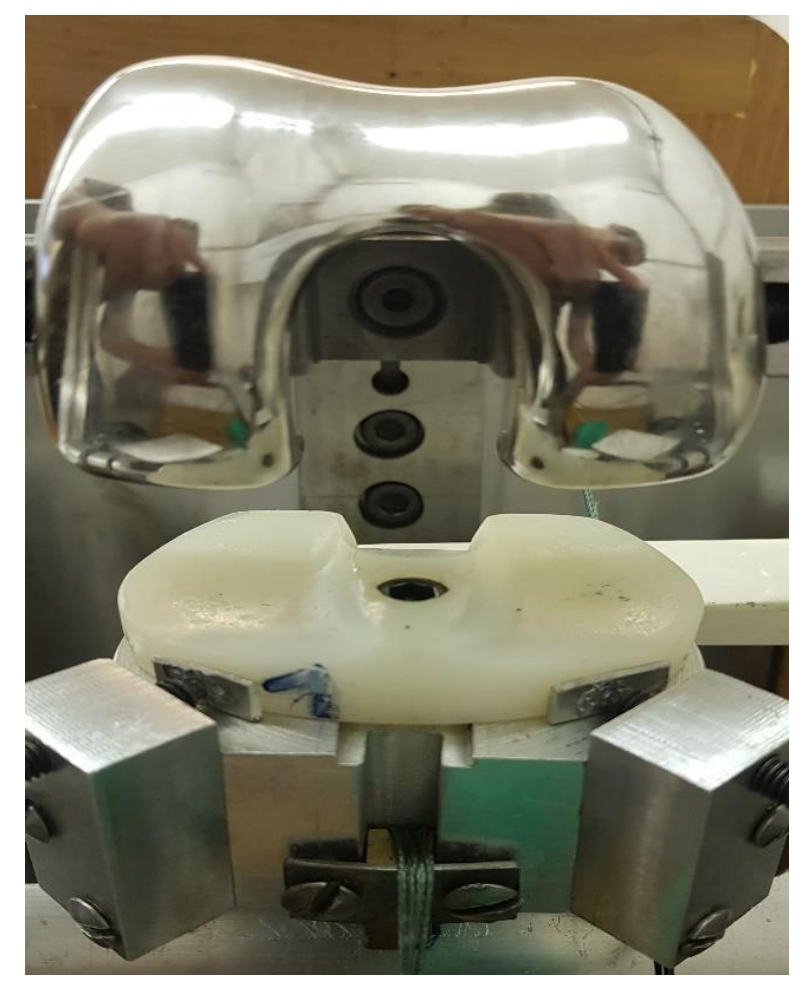

Figure 12. The tibia and the femoral component in the test machine

For the development of the geometry we use the ADAMS program, with the use of (ADAMS program) we optimize the tibia joint surfaces.

To perform the model of the knee prosthesis in ADAMS, in a first step, the 3D model of the knee prosthesis made in SolidWorks we will transfer it to the ADAMS database using the Para solid transfer interface. 


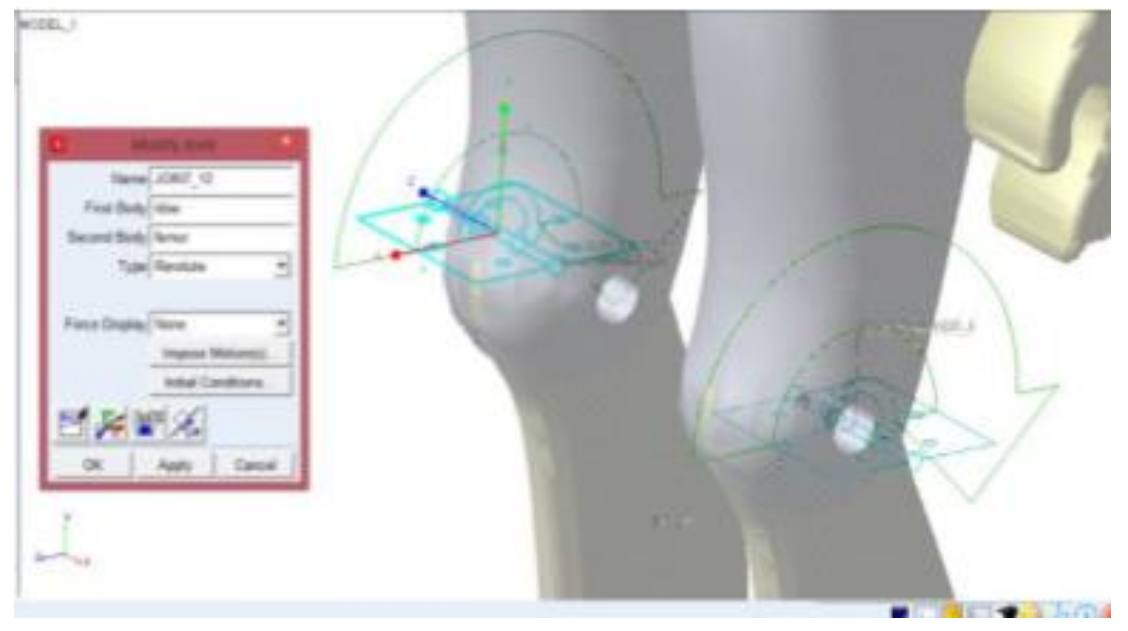

Figure 13. Design knee prosthesis in the program ADAMS, (D.Marian, 2017).

\section{Results}

According to the experimental for this system, many sets of results will obtain from this system to achieve the objectives.

\subsection{Virtual modeling of the knee prosthesis components Geometric modeling.}

To perform the multibody model of the virtual knee in ADAMS, at first we formed a simple form of a virtual knee, shown below

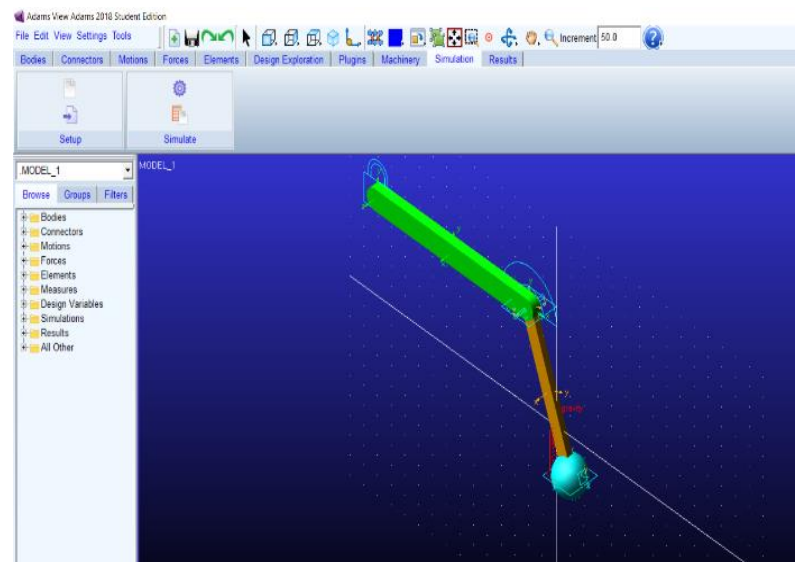

Figure 14. virtual modeling knee prosthesis from up

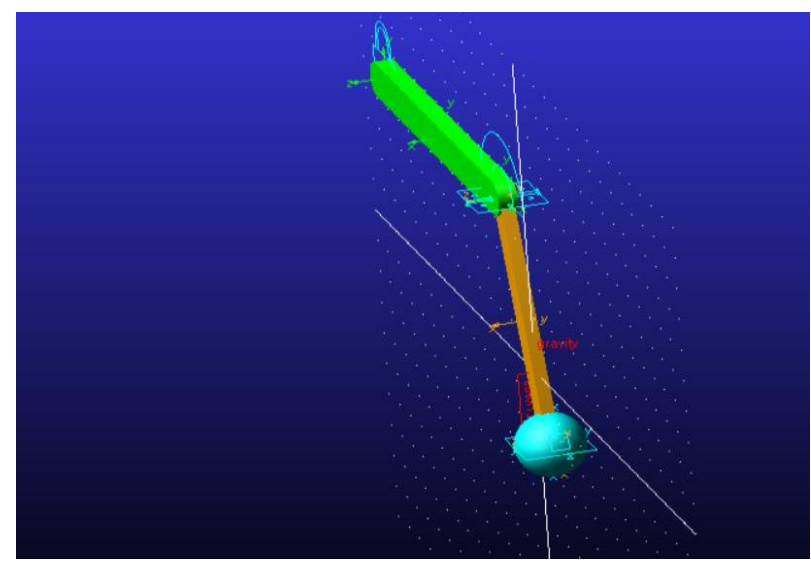

Figure 15. virtual modeling knee prosthesis from the front

\subsection{Results of the movement simulation}

The category of results obtained is the numerical results in the form of graphs of time variation of the kinematic or dynamic parameters of the virtual knee. Fig.14 shows the reactions obtained, The values 
of the ground reaction forces and their variation during the walking cycle are useful to compare them with the experimentally obtained results in order to validate the multibody model in ADAMS.

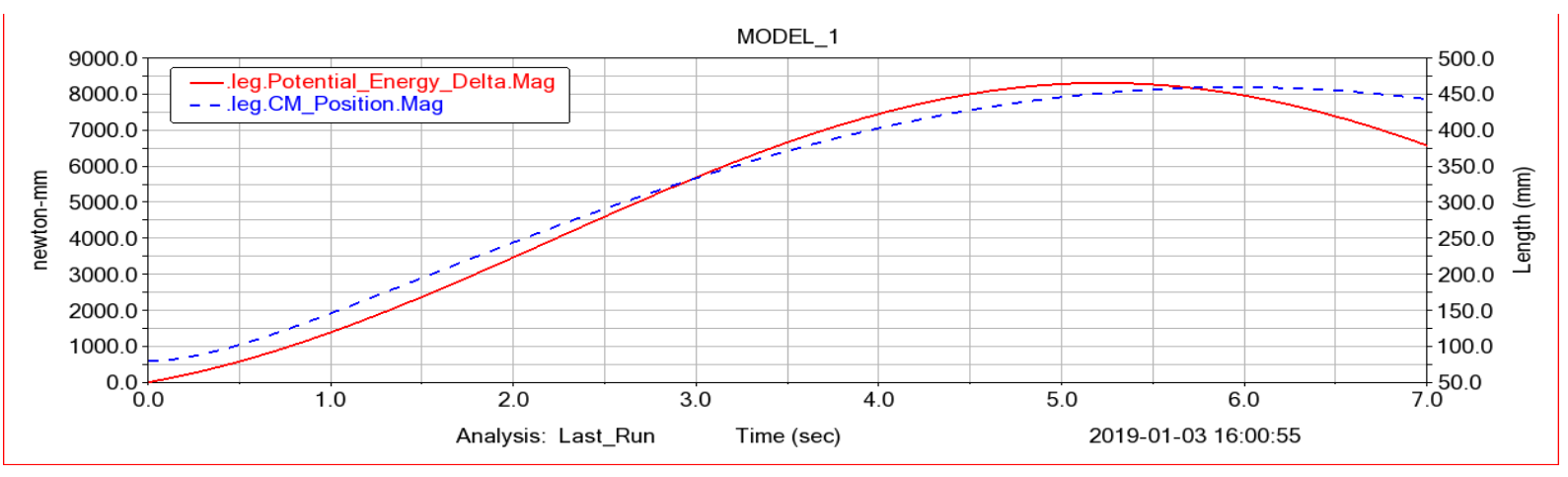

Figure 16.Contact forces (ground reactions) for the leg of the virtual knee.

\section{Conclusions}

Therefore, the results obtained by numerical in ADAMS for ground reaction forces are only prelimiary results, these aspects and conclusions validate the correctness of the virtual modeling of the knee and confirm the possibility of taking the forces from the joints for their use in various finite element analyses. So For the development of knee prosthesis I will use ADAMS program without (3D printing and $\mathrm{CNC}$ milling), until get the lower measurement and the fastest way a 3D model format.

\section{References}

[1] S. Standring (2008) Gray's Anatomy. The anatomical basis of clinical practice, New York: ChurchillL ivingstone: Elsevier Press, pp. 4300-4354.

[2] J. Bert (2005) Unicompartmental knee replacement. Orthopedic Clinics of North America, 36, pp. 512-22.

[3] N.E. Akalan, M. Ozkan, Y. Temelli (2008) Three-dimensional knee model: Constrained by isometric ligament bundles and experimentally obtained tibio-femoral contacts. Journal of Biomechanics. 41:890-6.

[4] J. Shi (2007) Finite element analysis of total knee replacement considering gait cycle load and malalignment. Doctor of Philosophy Thesis, University of Wolverhampton.

[5] D. Marian (2017) Research on the biomechanical study of the human knee joint with applications in prosthesis-PhD thesis, pp 61-65.

[6] G. Katona, B. M. Csizmadia, K. Andrónyi (2013) Determination of reference function to knee prosthesis rating. Biomechanica Hungarica, 6 (1), pp. 293- 301. 